\title{
Relaxation dynamics of the fastest channel in multichannel parallel relaxation mechanism is
}

\author{
Agnieszka Jurlewicz $^{\mathrm{a}, *}$, Karina Weron ${ }^{\mathrm{b}}$ \\ a Institute of Mathematics, Technical University of Wroclaw, Wyspiańskiego 27, 50-370 Wroclaw, Poland \\ ${ }^{\mathrm{b}}$ Institute of Physics, Technical University of Wroclaw, Wyspiańskiego 27, 50-370 Wroclaw, Poland
}

\begin{abstract}
Empirical evidence accumulated over the years shows that the time-dependent change of macroscopic properties of physical systems evolving to equilibrium exhibits a great degree of universality. We address the question of the origins of the universal relaxation laws in terms of probabilistic approach based on the multichannel parallel relaxation mechanism. We present a clear stochastic scheme uniquely leading to the whole class of experimentally observed relaxation responses. The proposed scheme results from the common assumption that only the fastest channel contributes to relaxation dynamics. (c) 1999 Elsevier Science Ltd. All rights reserved.
\end{abstract}

\section{Introduction}

The study of nonexponential relaxation is a problem of topical interest in nonequilibrium statistical physics. It is of importance in the study of a variety of problems from condensed matter physics [1,2], nuclear physics [3], spectroscopy [4,5], rheology [6], seismology [7], physical chemistry [8], molecular biophysics $[9,10]$, cell and population dynamics [11,12], etc. Among different relaxation functions suggested in the literature [1] the Kohlrausch-Williams-Watts (KWW) function

$$
\phi(t)=\exp \left(-(A t)^{\alpha}\right), \quad 0<\alpha \leqslant 1, A>0,
$$

plays an important role. Although the stretched exponential decay law (1.1) appears frequently enough to call attention for the origins of its ubiquity, it is not universally valid [13,14]. On the basis of linear dielectric response measurements, allowing one to follow the relaxation process over several decades of time, the existence of the two power-law response

$$
-\frac{\mathrm{d} \phi}{\mathrm{d} t} \propto \begin{cases}(A t)^{-n} & \text { for } A t \ll 1 \\ (A t)^{-m-1} & \text { for } A t \gg 1\end{cases}
$$

in relaxation dynamics of complex systems has been established unambiguously [14]. The parameters $0<n, m \leqslant 1$ and $A>0$ are constants characteristic to the material.

The wide occurence of the KWW stretched exponential relaxation (1.1), independently of the particular material properties, has attracted (and still does) much theoretical attention for the underlying reason of this phenomenon. It has been commonly assumed that the KWW function corresponds to a kind of universal

\footnotetext{
This work was partly supported by KBN Grant No 2 PO3B 10013.

${ }^{*}$ Corresponding author.

E-mail addresses: agniesz@im.pwr.wroc.pl (A. Jurlewicz), karina@rainbow.if.pwr.wroc.pl (K. Weron)
} 
behavior which is independent of the details of individual processes and this has stimulated the proposal of several "universal" mechanisms based either on parallel channel relaxation [14-19] or on hierarchically constrained dynamics [17,20,21]. In contrast, much less attention has been paid to the theoretical interpretation of the broader class (1.2) of relaxation responses. Let us note that the considerable difference between (1.1) and (1.2) is observed in the long-time limit where the time derivative of the KWW function does not exhibit the power-law behavior.

The purpose of this paper is to present a model which generates both the stretched exponential (with the special exponential case $\alpha=1$ ) and the two power-law responses. Since any relaxation process results from an appropriate transitional configuration in the system, imposed by nonequilibrium constraints at time $t=0$, and is conditioned by specific interactions of different parts of the system, both with local random characteristics, it is natural to address the question about the origins of the universal relaxation law in terms of probabilistic approach. In our opinion, in order to understand the universality of relaxation responses one needs to consider the nonexponential relaxation in a way that separates it from a particular physical context. Crucially relevant to this issue is the problem how to relate the local random characteristics of relaxing complex systems to the deterministic relaxation laws (1.1) and (1.2) observed on the macroscopic level. Such a relation has been proposed in a series of papers [22-31] devoted to stochastic modeling of relaxation phenomena via limit theorems of probability theory [32-36].

In this paper we study the so-called first passage of the system in which relaxation is due to events occuring through a collection of independent channels characterized by individual relaxation (transition) rates. The notion of the first passage as the survival probability of an initial nonequilibrium state of a complex system has been introduced $[23,24]$ to reflect the basic assumption taken in different approaches to relaxation phenomena [14-21] that only the fastest channel of relaxation contributes to relaxation dynamics.

\section{Survival probability as the first passage of a complex system}

The question of the origins of nonexponential relaxation addressed in terms of the parallel channel relaxation models is based on the distribution of random relaxation rates $\beta_{i}$, each corresponding to one channel of relaxation. The channels operate in a parallel (independent) ways and it is commonly assumed that only the fastest channel contributes to relaxation dynamics. According to different approaches to relaxation phenomena [14-31] the relaxation function $\phi(t)$ represents the probability $\operatorname{Pr}(\tilde{\theta} \geqslant t)$ that the transition of the system from its initial nonequilibrium state does not happen prior to a time instant $t$. Here $\tilde{\theta}$ denotes the life time of the initial state of the system as a whole. This probability can be given [23-31] by the first passage of the system defined as $\min \left(\theta_{1}, \ldots, \theta_{v_{N}}\right)$ where $\theta_{i}, i=1,2, \ldots N$, is the life time of the $i$ th initially imposed substate and $v_{N}$ is a number of substates taken into account (for example $v_{N}=N$ ). Namely,

$$
\phi(t)=\operatorname{Pr}(\tilde{\theta} \geqslant t)=\lim _{N \rightarrow \infty} \operatorname{Pr}\left(A_{N} \min \left(\theta_{1}, \ldots, \theta_{v_{N}}\right) \geqslant t\right)
$$

for a suitable sequence of normalizing constants $A_{N}$. Such a notion of the survival probability simply expresses the fact that only the fastest channel contributes to relaxation dynamics. The nonnegative $\theta_{1}, \theta_{2}, \ldots$ are assumed to form a sequence of independent and identically distributed random variables with a common probability distribution $F_{\theta}(t)$. By means of Extreme Value Theory [35] we obtain immediately that in case $v_{N}=N$ the only possible form of $\phi(t)$ defined by (2.1) is the stretched exponential function (1.1) but with any positive value of parameter $\alpha$ permitted. Note that experimental data give the restriction $0<\alpha \leqslant 1$.

Since every physical transformation process is conditioned by the interactions of different parts of the system (the causality principle $[1,17,21,37]$ ), the probability of preservation of the initial substate until a time instant $t$ must necessarily be conditioned by appropriate variates (in general, random) reflecting the influence of internal dynamics. In terms of the probability theory we can express it as [23-25]

$$
\operatorname{Pr}\left(\theta_{i} \geqslant t\right)=\int_{0}^{\infty} \operatorname{Pr}\left(\theta_{i} \geqslant t \mid \beta_{i}=b\right) w(b) \mathrm{d} b,
$$


where $w(b)$ is the probability density of the random variable $\beta_{i}$. It is natural to assume the exponential decay of an $i$ th nonequilibrium substate with its individual randomly distributed relaxation rate $\beta_{i}$ :

$$
\operatorname{Pr}\left(\theta_{i} \geqslant t \mid \beta_{i}=b\right)=\exp (-b t) \text { for } t \geqslant 0, b>0 .
$$

The nonnegative random relaxation rates $\beta_{i}, i=1,2 \ldots$, form a sequence of independent and identically distributed random variables. Since Eq. (2.1) can be rewritten as

$$
\phi(t)=\lim _{N \rightarrow \infty} \operatorname{Pr}\left(\theta_{1} \geqslant \frac{t}{A_{N}}\right) \ldots \operatorname{Pr}\left(\theta_{v_{N}} \geqslant \frac{t}{A_{N}}\right)=\lim _{N \rightarrow \infty}\left(\operatorname{Pr}\left(\theta_{1} \geqslant \frac{t}{A_{N}}\right)\right)^{v_{N}}
$$

the relaxation function (2.1) can be expressed also as

$$
\phi(t)=\lim _{N \rightarrow \infty}\left\langle\exp \left(-t \sum_{i=1}^{v_{N}} \beta_{i} / A_{N}\right)\right\rangle=\left\langle\exp \left(-t \tilde{\beta}^{*}\right)\right\rangle,
$$

where $\langle\cdot\rangle$ denotes the expected (mean) value and $\tilde{\beta}^{*}$ is a limit in distribution of the normalized sums $\sum_{i=1}^{v_{N}} \beta_{i} / A_{N}$

$$
\tilde{\beta}^{*}=\lim _{N \rightarrow \infty} \sum_{i=1}^{v_{N}} \beta_{i} / A_{N} .
$$

The limit theorem (2.3) indicates that the transition probability per unit time $\tilde{\beta}^{*}$ (the effective relaxation rate) for the system as a whole is equal to a normalized sum of probabilities per unit time $\beta_{i}$ over all $v_{N}$ possible routes for its realization. This is in agreement with the common assumption in multichannel parallel relaxation models [14-19,37] that a transformation of a complex system can be realized in several independent ways. It also follows from (2.2) that the relaxation function $\phi(t)$ defined in (2.1) simply expresses the idea of the historically oldest probabilistic attempt to relaxation [1] in which $\phi(t)$ has been defined as a weighted average of exponential decay with respect to the relaxation rate distribution $F_{\tilde{\beta}^{*}}(b)$. Namely, the expected value of the random variable $\exp \left(-t \tilde{\beta}^{*}\right)$ in $(2.2)$ can be written explicitly as

$$
\phi(t)=\int_{0}^{\infty} \exp (-b t) \mathrm{d} F_{\tilde{\beta}^{*}}(b) .
$$

It should be noted that we can come to the same conclusions even if the number $v_{N}$ in definition (2.1) is not a deterministic number but an integer-valued random variable (independent of the life times $\theta_{i}$ and relaxation rates $\beta_{i}$ ) the distribution of which depends on $N$.

It has been shown [25] that in case of deterministic number $v_{N}=N$ the only possible probability distributions $F_{\tilde{\beta}^{*}}(b)$ for the effective relaxation rate $\tilde{\beta}^{*}=\tilde{\beta}$ are completely asymmetric Lévy-stable laws $S_{\alpha}(b)$ with the parameter $0<\alpha<1$ together with degenerate case $\tilde{\beta}=$ const obtained when $\alpha \rightarrow 1$. These distributions are easily defined by their Laplace transforms:

$$
\mathscr{L}(\tilde{\beta}, t)=\int_{0}^{\infty} \exp (-b t) \mathrm{d} S_{\alpha}(b)=\exp \left(-(A t)^{\alpha}\right)
$$

where $A$ is a positive scale constant. Consequently, the only possible formula for the relaxation function (2.1) is the KWW form (1.1).

Allowing the number $v_{N}$ in definition (2.1) to be random one can broaden the class of possible relaxation functions according to the class of probability distributions used to describe this randomness. For example, assuming that $v_{N}$ is distributed geometrically, i.e.,

$$
\operatorname{Pr}\left(v_{N}=n\right)=\frac{1}{N}\left(1-\frac{1}{N}\right)^{n-1}, \quad n=1,2, \ldots
$$

one obtains the effective relaxation rate $\tilde{\beta}^{*}$ distributed according to the so-called geo-stable law that leads to form the relaxation function 


$$
\phi(t)=\frac{1}{1+(A t)^{\alpha}}, \quad 0<\alpha \leqslant 1,
$$

that exhibits two power-law behavior (1.2) with $n=1-\alpha$ and $m=\alpha$. Such a response concerns only a small class of experimental data fitted with the Cole-Cole empirical function [1]. It is worth noting that the law of the effective relaxation rate can be represented in this case as a mixture of one-sided Lévy-stable and the exponential distributions. Namely,

$$
\tilde{\beta}^{*}=E^{1 / \alpha} \tilde{\beta}
$$

where $E$ is exponentially distributed random variable (with the probability density function $g(\lambda)=\exp (-\lambda)$ for $\lambda>0$ ) which is independent of the Lévy-stable effective relaxation rate $\tilde{\beta}$ obtained in case of deterministic number $v_{N}$. The probability distribution $F_{\tilde{\beta}^{*}}(b)$ of effective relaxation rate $\tilde{\beta}^{*}$ gets then the integral form

$$
F_{\tilde{\beta}^{*}}(b)=\int_{0}^{\infty} S_{\alpha}\left(\frac{b}{\lambda^{1 / \alpha}}\right) g(\lambda) \mathrm{d} \lambda .
$$

This type of mixture has been shown to produce the Mittag-Leffler law [38] and hence

$$
F_{\tilde{\beta}^{*}}(b)=\sum_{i=0}^{\infty} \frac{(-1)^{i}}{\Gamma(1+\alpha(1+i))}\left(\frac{b}{A}\right)^{\alpha(1+i)} .
$$

Considering the class of negative binomial [32] distributions for $v_{N}$ (which are in fact a generalization of geometrical law) one can get [31] the following form of the relaxation function:

$$
\phi(t)=\frac{1}{\left(1+k(A t)^{\alpha}\right)^{1 / k}}, \quad 0<\alpha \leqslant 1, k>0,
$$

that in case $k \geqslant \alpha$ exhibits two power-law behavior (1.2) with $n=1-\alpha$ and $m=\alpha / k$. Observe that when $k=1$ the relaxation function (2.5) coincides with (2.4) while $k \rightarrow 0$ leads to the KWW function (1.1). In general, the derived form (2.5) corresponds to the whole class of response functions observed in dielectric relaxation phenomena.

The effective relaxation rate $\tilde{\beta}^{*}$ leading to $(2.5)$ is distributed according to the negative-binomial-stable law that can be recognized as the generalized Mittag-Leffler distribution [38]

$$
F_{\tilde{\beta}^{*}}(b)=\sum_{i=0}^{\infty} \frac{(-1)^{i} \Gamma(i+1 / k)}{\Gamma(1 / k) i ! \Gamma(1+\alpha(1 / k+i))}\left(\frac{b}{A k^{1 / \alpha}}\right)^{\alpha(1 / k+i)} .
$$

Thus, it can be represented as

$$
\tilde{\beta}^{*}=\left(k \Gamma_{k}\right)^{1 / \alpha} \tilde{\beta}
$$

where $\Gamma_{k}$ is a random variable distributed according to the gamma law, with the probability density function

$$
g_{k}(\lambda)=\frac{1}{\Gamma(1 / k)} \lambda^{1 / k-1} \exp (-\lambda) \text { for } \lambda>0
$$

which is independent of the Lévy-stable effective relaxation rate $\tilde{\beta}$ obtained in deterministic case. Hence

$$
F_{\tilde{\beta}^{*}}(b)=\int_{0}^{\infty} S_{\alpha}\left(\frac{b}{(k \lambda)^{1 / \alpha}}\right) g_{k}(\lambda) \mathrm{d} \lambda .
$$

In general, it is possible to obtain in this way any $v$-stable effective relaxation rate by proper choice of a distribution of $v_{N}$. The representation of the distribution of $v$-stable random variables via Lévy-stable laws, as well as its tail properties has been studied recently [36]. 


\section{Concluding remarks}

In order to understand the universal relaxation behavior, which is independent of the details of individual processes, we have proposed the stochastic model based on the universal mechanism hidden behind the limit theorems of probability theory. The notion of the first passage of a complex system has been introduced, according to the basic assumption taken in different approaches to relaxation phenomena, that only the fastest channel of relaxation contributes to dynamics of this process. The relaxation function defined as a survival probability of an initial nonequilibrium state of the system has been expressed by means of the first passage of the system, and then shown to have the form (2.5) with the KWW function, $0<\alpha<1$, and the classical exponential decay as limiting cases. The effective relaxation rate $\tilde{\beta}^{*}$ responsible for the macroscopic behavior of the relaxing system has been shown to have the form (2.3) indicating that a transformation of a complex system is realized in several independent ways. We have observed that the existence of the effective relaxation rate in (2.3), as well as its probability distribution $F_{\tilde{\beta}^{*}}(b)$, directly follow from the limit theorems of probability theory and uniquely determine the form of the relaxation function. Using the results of the limit theorems for summation scheme with deterministic and random number of summands, we have derived three forms of the relaxation function (2.1), namely

- the classical exponential decay form corresponding to the nonrandom effective relaxation rate, i.e., $\tilde{\beta}=$ const with probability 1 ;

- the KWW form (1.1) corresponding to the effective relaxation rate distributed according to the one-sided Lévy-stable probability distribution (obtained in the case of deterministic number of summands);

- the form (2.5) corresponding to the effective relaxation rate distributed according to the Mittag-Leffler probability distribution (obtained in the case of random, negative binomially distributed number of summands).

\section{References}

[1] A.K. Jonscher, Dielectric Relaxation in Solids, Chelsea Dielectrics Press, London, 1983.

[2] S. Dattagupta, Relaxation Phenomena in Condensed Matter, Physics, Akademic Press, Orlando, 1987.

[3] F.M. Dittes, H.L. Harney, A. Műller, Phys. Rev. A 45 (1992) 701.

[4] A.G. Kofman, R. Zaibel, A.M. Levine, Y. Prior, Phys. Rev. A 41 (1990) 6434.

[5] M.O. Vlad, Int. J. Modern Phys. B 7 (1993) 2539.

[6] T.F. Nonnenmacher, in: Rheological Modeling: Thermodynamical and Statistical Approaches, Lecture Notes in Physics, vol. 381, Springer, Berlin, 1991, p. 309.

[7] J. Koyama, H. Hara, Phys. Rev. A 46 (1992) 1844.

[8] A. Ptonka, Time-Dependent Reactivity of Species in Condensed Media, Lecture Notes in Chemistry, vol. 40, Springer, Berlin, 1986.

[9] R. Zwanzig, J. Chem. Phys. 97 (1992) 3587.

[10] J. Wang, P. Wolynes, Chem. Phys. 180 (1994) 141.

[11] A. Lasota, M.C. Mackey, J. Math. Biol. 10 (1980) 333.

[12] M.C. Mackey, J. Milton, J. Math. Biol. 28 (1990) 33.

[13] S. Havriliak Jr., S.J. Havriliak, J. Non-Cryst. Solids 172-174 (1994) 297.

[14] A.K. Jonscher, Universal Relaxation Law, Chelsea Dielectrics Press, London, 1996.

[15] A. Blumen, Nuovo Cimento B 63 (1981) 50.

[16] E.W. Montroll, J.T. Bendler, J. Statist. Phys. 34 (1984) 129.

[17] J. Klafter, M.F. Shlesinger, Proc. Natl. Acad. Sci. USA 83 (1986) 848.

[18] I. Koponen, J. Non-Cryst. Solids 189 (1995) 154.

[19] M.O. Vlad, M.C. Mackey, J. Math. Phys 36 (1995) 1834.

[20] L.A. Dissado, R.M. Hill, Proc. Roy. Soc. A 390 (1983) 131.

[21] R.G. Palmer, D. Stein, E.S. Abrahams, P.W. Anderson, Phys. Rev. Lett. 53 (1984) 958.

[22] K. Weron, Acta Phys. Pol. A 70 (1986) 529.

[23] K. Weron, J. Phys.: Condens. Matter 3 (1991) 9151.

[24] K. Weron, J. Phys.: Condens. Matter 4 (1992) 10507.

[25] K. Weron, A. Jurlewicz, J. Phys. A 26 (1993) 395.

[26] A. Jurlewicz, K. Weron, J. Statist. Phys. 73 (1993) 69.

[27] A. Jurlewicz, J. Stat. Phys. 79 (1995) 993.

[28] K. Weron, K. Kosmulski, A. Jurlewicz, S. Mercik, in: Chaos - The Interplay between Stochastic and Deterministic Behaviour, Lecture Notes in Physics, vol. 457, Springer, Berlin, 1995, p. 545. 
[29] A. Weron, K. Weron, W.A. Woyczynski, J. Statist. Phys. 78 (1995) 1027.

[30] A. Jurlewicz, A. Weron, K. Weron, Appl. Math. 23 (1996) 379.

[31] K. Weron, M. Kotulski, J. Statist. Phys. 88 (1997) 1241.

[32] W. Feller, An Introduction to Probability and Its Applications, vol. 2, Wiley, New York, 1966.

[33] V.M. Zolotariev, One-dimensional Stable Distributions, American Mathematical Society, Providence, RI, 1986.

[34] A. Janicki, A. Weron, Simulation and Chaotic Behavior of $\alpha$-Stable Stochastic Processes, Marcel Dekker, New York, 1993.

[35] M.R. Leadbetter, G. Lindgren, H. Rootzen, Extremes and Related Properties of Random Sequences and Processes, Springer, New York, 1986.

[36] T.J. Kozubowski, A.K. Panorska, Statist. Prob. Lett. 29 (1996) 307.

[37] L. Mayants, The Enigma of Probability in Physics, Reidel, Dordrecht, 1984.

[38] R.N. Pillai, Ann. Inst. Statist. Math. 42 (1990) 157. 